\title{
Extending opportunities
}

\author{
Innovative mid-infrared laser technologies are anticipated to broaden the applications of existing mid- \\ infrared laser sources and bring unexpected scientific discoveries.
}

Spanning the wavelength range of $2-20 \mu \mathrm{m}$ $\left(500-5,000 \mathrm{~cm}^{-1}\right)$, the mid-infrared (mid-IR) is a spectral region of tremendous scientific and technological interest. It contains the strong characteristic vibrational transitions of many important molecules, making mid-IR laser sources crucial for applications in spectroscopy, materials processing and chemical and biomolecular sensing. The two atmospheric transmission windows of 3-5 $\mu \mathrm{m}$ and $8-13 \mu \mathrm{m}$, in which the Earth's atmosphere is relatively transparent, are also important for atmospheric, security and industrial applications such as remote explosive detection, countermeasures against heat-seeking missiles and covert communication systems.

Light sources operating at near-IR and visible wavelengths have developed steadily into commercially available instruments. Developments in mid-IR laser source technology, however, have faced considerable challenges. A number of different approaches are currently available, each offering its own range of features, operating standard and wavelength range. For instance, semiconductor lasers, optically pumped semiconductor lasers and optical parametric oscillators are readily available and are now becoming more compact and robust. Meanwhile, many newly emerging mid-IR laser technologies are anticipated to expand the applications of mid-IR laser sources.

This issue of Nature Photonics features a special focus on mid-IR photonics, covering recent developments in sophisticated laser technologies such as quantum cascade lasers, fibre lasers and frequency combs, and discussing how to exploit plasmonics in midIR photonics.

Quantum cascade lasers currently form the basis of mid-IR photonics and are a relatively recent development in the field of semiconductor lasers. Unlike earlier mid-IR semiconductor lasers based on interband transitions, quantum cascade lasers utilize intersubband transitions within a multiplequantum-well structure. This offers excellent design flexibility because the staircase of intersubband transitions can be designed to obtain particular emission wavelengths. With the recently achieved tuning range of 3-25 $\mu \mathrm{m}$, quantum cascade lasers are rapidly becoming practical mid-IR sources for a wide variety of applications, such as trace-

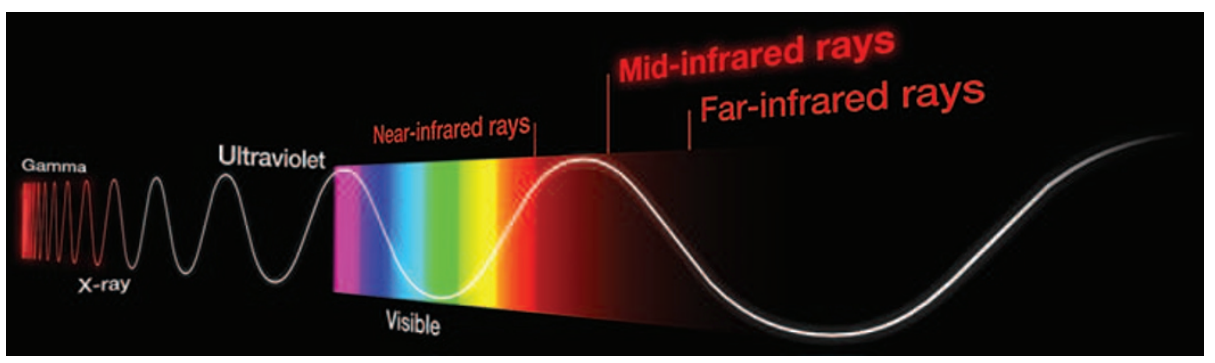

chemical sensing, health monitoring and IR countermeasures.

In a Review on page 432, Yu Yao, Anthony Hoffman and Claire Gmachl discuss the recent progress in this field particularly recent improvements in power, power efficiency and spectral performance. The researchers point out fundamental issues related to the development of a subpicosecond, high-pulse-energy pulsed quantum cascade laser and the development of material systems that are very different from conventional group III-V semiconductor alloys. They also discuss the challenges of achieving power specifications that are suitable for consumer electronics.

On page 423, Stuart Jackson reviews the recent advances and future challenges of midIR fibre lasers. The gain medium in a fibre laser is an optical fibre doped with rare-earth elements such as thulium, erbium, ytterbium or dysprosium. The choice of doping element is important because its fluorescence transitions determine the device's lasing wavelength. Host materials such as silicate, fluoride and ZBLAN provide the necessarily high transmission and sufficient radiative efficiency for the rare-earth cation transitions.

Rapid developments in fabrication facilities have provided enormous flexibility in the characteristics and quantity of light that can be generated from a fibre laser. For instance, the maximum continuouswave output power available from a diodepumped silicate fibre laser emitting at around $2 \mu \mathrm{m}$ has increased by three orders of magnitude over the past two decades, making it attractive for cutting and welding applications in the automotive industry. However, Jackson points out that fibre lasers experience a clear exponential decrease in maximum continuous-wave output power as the wavelength is increased, regardless of the particular doping cation or host material.
The realization of fibre lasers capable of emitting beyond $3 \mu \mathrm{m}$ and achieving long-term reliability of future multikilowatt-level $2 \mu \mathrm{m}$ fibre laser systems are significant challenges.

Frequency combs are coherent light sources that emit a broad spectrum of discrete, evenly spaced narrow lines. The absolute frequency of these lines can be measured to within the accuracy of an atomic clock, which makes frequency combs useful devices for calibrating astronomical spectrographs and molecular spectroscopy. In a Review on page 440, Albert Schliesser, Nathalie Picqué and Theodor Hänsch discuss innovative solutions based on novel laser gain media, nonlinear frequency conversion and Kerr comb generators covering vast ranges of repetition frequency and spectral span. They also highlight some of the field's most important goals, including direct laser emission of few-cycle phase-coherent pulses, improved materials for efficient nonlinear frequency conversion over a broad spectral bandwidth beyond $6 \mu \mathrm{m}$, and octavespanning low-phase-noise microresonatorbased frequency combs centred deep in the mid-IR.

In a Commentary on page 409, Ross Stanley discusses how plasmonics can be employed to aid the field of mid-IR photonics. First studied in the visible region, plasmons charge oscillations at the interface between a dielectric and a metal - are now being exploited to enhance the performance of mid-IR sources, sensors and detectors for applications such as chemical sensing, thermal imaging and heat scavenging. According to Stanley, a better understanding of the interplay between geometrical and material impedance is the key to success. The challenge now is to integrate mid-IR plasmonics technologies in cost-effective, compact and reliable platforms. 